\title{
Inguinal Hernia with Intestinal and Ovarian Content: A Rare Case in Pediatric
} Age

\author{
Sonia Tamasi ${ }^{1}$ | Rocco Minelli2* $^{2 *}$ Maria G Giagnorio $^{2}$ | Eugenio Rossi ${ }^{3}$ | Rosanna Mamone ${ }^{1}$ | Dolores \\ Ferrara $^{1}$ | Donatella Irace ${ }^{4}$ | Elio Bignardi ${ }^{5}$ | Sandra Coppola ${ }^{6} \mid$ Silvana Russo $^{6}$ | Mattia Carbone $^{7}$ | Massimo \\ Zeccolini $^{1}$
}

*Correspondence: Rocco Minelli

Address: ${ }^{1}$ U.O.C. of General Radiology P.O. Santobono-A.O.R.N. Santobono-Pausilipon, Via M. Fiore 6, 80128, Naples, Italy; 2Department Life and Health "V. Tiberio", University of Molise, Via Francesco De Sanctis 1, Campobasso 86100, Italy; ${ }^{3}$ U.O.S.D. Radiology and Ultrasound P.O. Pausilipon-AORN Santobono-Pausilipon, Via Posillipo 226, 80123 Naples, Italy; ${ }^{4}$ A.O.U. Federico II, Department of Translational Medical Sciences, Pediatric Section, Via S. Pansini, 5, Naples, Italy; ${ }^{5}$ Department of Radiology Cotugno Hospital, Naples, Italy; ${ }^{6}$ Pediatric Surgery Department P.O Santobono-A.O.R.N. Santobono-Pausilipon, Via M. Fiore 6, 80128, Naples, Italy; 7Department of Radiology, San Giovanni E Ruggi D’Aragona Hospital, Hospital, Via San Leonardo, Salerno, Italy

e-mail $\bowtie$ : rocco.minelli@libero.it

Received: 02 June 2020; Accepted: 11 June 2020

Copyright: (C) 2020 Tamasi S. This is an open-access article distributed under the terms of the Creative Commons Attribution License, which permits unrestricted use, distribution, and reproduction in any medium, provided that the original work is properly cited.

\section{ABSTRACT}

Inguinal hernia is a common congenital abnormality in children. Clinically it manifests itself with a palpable swelling in the groin. The ultrasound examination is essential to confirm the clinical hypotheses. We describe a rare case of inguinal hernia with both intestinal and ovarian contents in an infant who arrived at the emergency room of the "SantobonoPausilipon" pediatric hospital.

Keywords: Inguinal Hernia, Ovary, Intestine, Ultrasound

\section{Introduction}

Inguinal hernia is the most common form of hernia of the lower abdominal wall in children. The estimated incidence is between $0.8 \%$ and $4.4 \%$, occurs mainly in the first year of life, with greater frequency in males and a ratio between males and females of 6: 1 (Dan Poenaru, 2000). The inguinal hernia is to be considered a congenital anomaly secondary to a failure to obliterate the vaginal process. The hernial sac can contain intestinal loops, omentum and genital organs; the incidence of ovarian hernias is around 15-20\% in female infants (Laing et al., 2007). Rarer, however, are hernias containing the uterus and ipsilateral ovary or both ovaries (Cascini et al., 2013). On physical examination, the inguinal hernia appears as a palpable swelling, momentarily reducible, which can face complications such as incarceration. For this reason, early diagnosis and therapy are essential. Ultrasound and color 
Doppler are fundamental tools for diagnostic confirmation and for the evaluation of possible complications (Dal Mo Yang et al., 2014).

\section{Case Report}

A month-old infant arrived at the emergency room for the appearance of a sudden swelling in the left inguinal-crural site. Upon physical examination, the abdomen appeared treatable and painless on palpation. The swelling was partially reducible and reappeared with tears. In the suspicion of an inguinal hernia, an ultrasound examination was performed which showed a dehiscence of the muscle structures with engagement in the inguinal canal of intestinal loops (Fig. 1) and

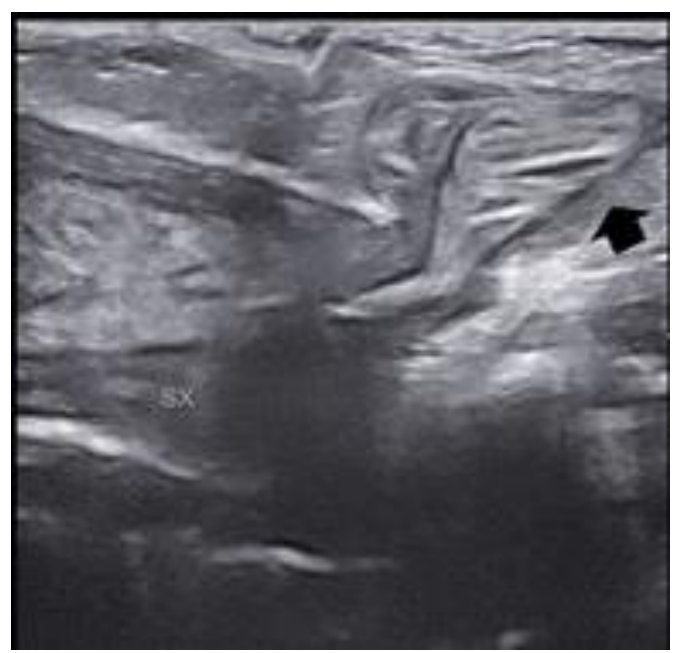

Figure 1: Longitudinal sonogram shows in correspondence with the swelling clinically palpable a dehiscence of the muscle structures with commitment in the inguinal canal of bowel loops (black arrow)

just below the intestinal loops, above the muscle wall on the clinically palpable swelling left side, a formation was evident, with an echogenic oval morphology with contextual cystic areolas, compatible with the annexal structure (Fig. 2).

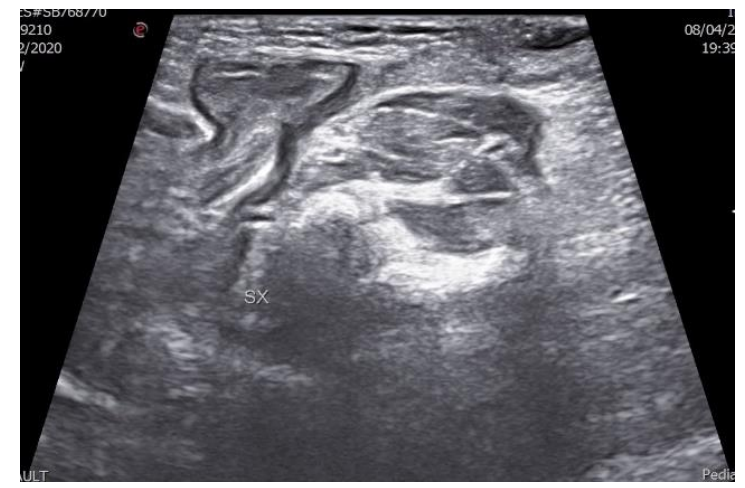

Figure 2: Longitudinal ultrasound scan showing double herniation 
On the eco-color Doppler examination, there was a regular vascularization of both the intestinal walls and the herniated ovary, thus excluding complications in progress (Fig. 3-4).

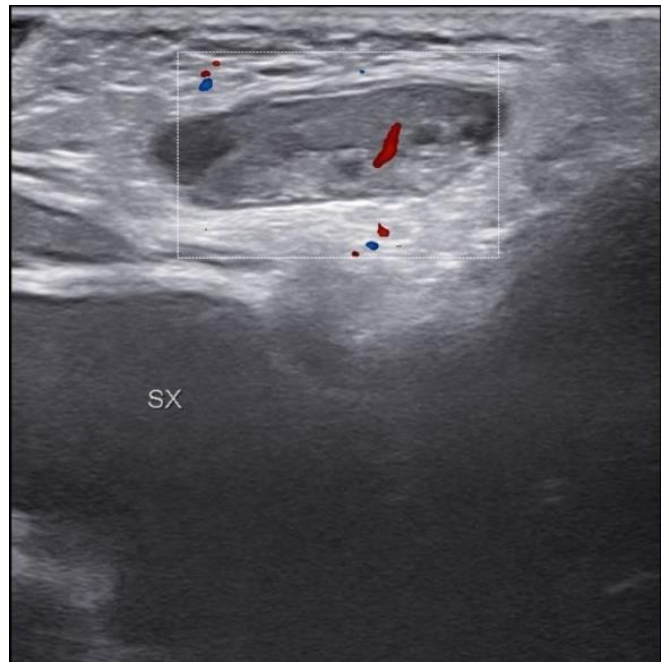

Figure 3: Longitudinal ultrasound scan with color-Doppler integration shows an echogenic oval formation with contextual cystic areolae compatible with adnexal structure

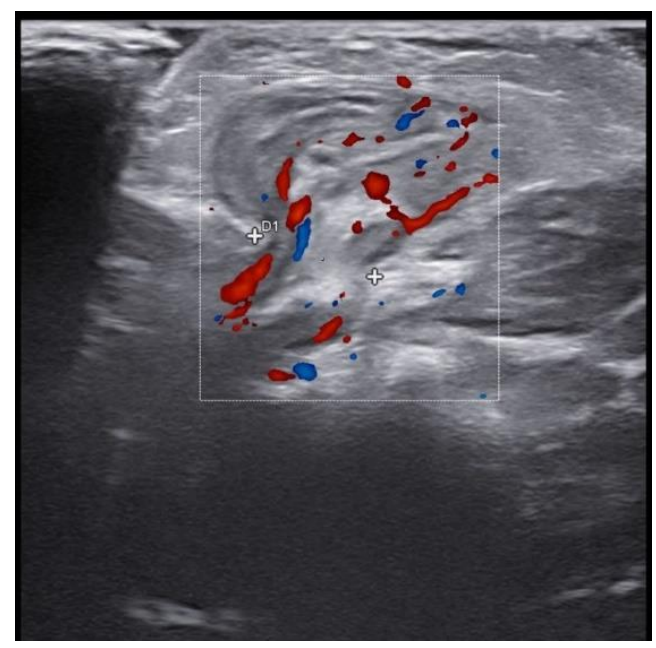

Figure 4: Longitudinal ultrasound scan with color-Doppler integration shows regular vascularization of both the intestinal walls and the herniated ovary

The infant was subjected to surgery, which confirmed the preoperative diagnosis.

\section{Discussion}

Inguinal hernia is a very frequent congenital anomaly in children and is secondary to changes in the inguinal canal that occur during embryonic development (Shadbolt et al., 2001). This development, in both the male and female foetuses, requires a series of phases involving two main anatomical structures: the gubernaculum testis and the vaginal process. The gubernaculum testis is a ligamentous 
formation that inserts above the lower pole of the fetal gonad and below the skin of the inguinal region (Merriman and Auldist, 2000). In the male foetus it helps the descent of the testicle through the inguinal canal into the scrotum while in the female foetus it prevents the ovary from descending into the inguinal canal. The vaginal process, called the Nuck canal in the female gender, is a peritoneal fold that invagines into the inguinal canal and, depending on the gender, accompanies the testicle or the round ligament of the uterus towards the scrotum or labia majora. At birth or shortly before the vaginal process obliterates; otherwise, a herniation of the intestinal loops or genital organs in the inguinal canal may occur. Therefore, a premature birth before the closure of this canal occurs increases the risk of developing an inguinal hernia (Ziegler, 1994). In about 60\% of cases, inguinal hernias arise on the right side because the right vaginal process obliterates later than the left; in $10 \%$ of cases the hernia can be bilateral (Ming et al., 2011). Inguinal hernias can have a varied content; the hernial sac may in fact contain intestinal loops, omentum, testes, ovaries, fallopian tubes, uterus and urinary bladder (Minella et al., 2020). On physical examination, uncomplicated hernias usually present with intermittent swelling in the groin region, which in males can extend to the scrotum and in females it can extend to the labia majora (Shalev et al., 2001). Swelling is generally reducible with light pressure but may recur after the reduction maneuver or during crying. Incarceration of inguinal hernias is an important complication in children and occurs with a frequency of $31 \%$. The intestine, the ovaries and the fallopian tubes are the organs that can most commonly encounter this complication that clinically manifests itself with nonreducible swelling (Caprio et al., 2019). Some hernias can regress spontaneously, less likely if the hernia has an ovarian content; therefore, even in the absence of complications, hernias with an ovarian content must be managed with early surgery. An incarcerated hernia can progress rapidly towards strangulation which leads to vascular impairment of the incarcerated contents. This is clinically manifested by abdominal pain, irritability and vomiting. The imprisoned ovaries are, in turn, susceptible to twisting (Rossi et al., 2019). Therefore, early diagnosis and therapy are essential to prevent irreversible damage to herniated structures. Imaging plays a fundamental role in the diagnostic framework of inguinal hernias (Tufano et al., 2020). In particular, ultrasound is the main diagnostic test, being an easily available, low cost and free of ionizing radiation exam. The ultrasound, in addition to giving a diagnostic confirmation, allows to recognize the hernial content through the characteristic ultrasound aspect of the anatomical structures involved and to preclude early the appearance of complications, which in the case of intestinal loops are announced by the thickening of the walls, by the presence of free fluid in the hernial sac and by intra-abdominal intestinal dilatation. In the event that the herniation has an ovarian content and there is a suspicion of torsion, it is possible to find a heterogeneous echogenic mass larger than $5 \mathrm{~cm}$, with follicles arranged peripherally (Draghi et al., 2020). No vascular signal appears on color-Doppler examination (Calle-Toro et al., 2019). Ultrasound examination should evaluate both inguinal channels, as clinically not evident contralateral hernia can be 
found in $88 \%$ of cases (Aso et al., 2005).

\section{Conclusion}

In the presence of an inguinal swelling in pediatric inguinal hernias must be considered in the differential diagnosis. Ultrasound should be used as a first choice imaging exam because it is essential for both diagnostic confirmation and evaluation of complications.

\section{Conflict of Interest}

The Authors declare that they have no conflict of interest.

\section{Informed Consent}

All procedures followed were in accordance with the ethical standards of the responsible committee on human experimentation (institutional and national) and with the Helsinki Declaration of 1975, and its late amendments. Additional informed consented was obtained from all patients for which identifying information is not included in this article.

\section{Human and Animal Rights}

This article does not contain any studies with human or animal subjects performed by any of the Authors.

\section{References}

Aso C, Enríquez G, Fité M, Torán N, Piró C, Piqueras J, Lucaya J. Gray-scale and color Doppler sonography of scrotal disorders in children: An update. Radiographics 2005; 25: 1197-1214.

Calle-Toro JS, Kelly A, Ford EJ, Zemel BS, Schall JI, Adgent MA, Umbach DM, Rogan WJ, Stallings VA, Darge K, Kaplan SL. Incidental findings during ultrasound of thyroid, breast, testis, uterus and ovary in healthy term neonates. J Ultrasound 2019; 22: 395-400.

Caprio MG, Di Serafino M, De Feo A, Guerriero E, Perillo T, Barbuto L, Vezzali N, Rossi E, Ferro F, Vallone G, Orazi C. Ultrasonographic and multimodal imaging of pedi-atric genital female diseases. J Ultrasound 2019; 22: 273-289.

Cascini V, Lisi G, Renzo DD, Pappalepore N, Chiesa PL. Irreducible indirect inguinal hernia containing uterus and bilateral adnexa in a premature female infant: Report of an exceptional case and review of the literature. J Pediatr Surg 2013; 48: e17e19

Dal Mo Yang, Hyun Cheol Kim, Sang Won Kim, Sung Jig Lim, Seung Jin Park, Joo Won Lim. Ultrasonographic diagnosis of ovary-containing hernias of the canal of Nuck. Ultrasonography 2014; 33: 178-183 
Dan Poenaru. Inguinal hernias and hydroceles in infancy and childhood: A consensus statement of the Canadian Association of Paediatric Surgeons. Paediatr Child Health 2000; 5: 461-462.

Draghi F, Cocco G, Richelmi FM, Schiavone C. Abdominal wall sonography: a pictorial review. J Ultrasound 2020; 3: 1-4.

Laing FC, Townsend BA, Rodriguez JR. Ovarycontaining hernia in a premature infant: Sonographic diagnosis. $J$ Ultrasound Med 2007; 26: 985-987

Merriman TE and Auldist AW. Ovarian torsion in inguinal hernias. Pediatr Surg Int 2000; 16: 383-385

Minella R, Minelli R, Rossi E, Cremone G, Tozzi A. Tozzi. Gastroesophageal and gastric ultrasound in children: the state of the art. Journal Ultrasound, 2020.

Ming Y, Luo C, Chao HC, Chu SM. Inguinal hernia containing uterus and uterine adnexa in female infants: Report of two cases.Pediatr. Neonatol 2011; 52: 103-105

Rossi E, Minelli R, Di Serafino M, Brillantino C, Bignardi E, Vezzali N, Vallone G, Zeccolini M. Ultrasound assessment of peritoneal and retro-peritoneal masses in paediatric age: a pictorial essay. Il Giornale Italiano di Radiologia Medica ${ }^{\circ} 167$ Maggio-Giugno 2019; 6: 259-267.

Shadbolt CL, Heinze SB, Dietrich RB. Imaging of groin masses: inguinal anatomy and pathologic conditions revisited. Radiographics 2001; 21: 261-271

Shalev J, Mashiach R, Bar-Hava I, Girtler O, Bar J, Dicker D, Meizner I. Subtorsion of the ovary: Sonographic features and clinical management. J Ultrasound Med 2001; 20: 849-854

Tufano A, Minelli R, Rossi E, Brillantino C, Di Serafino M, Zeccolini M, Cantisani V, Vallone G. Inferior epigastric artery pseudoaneurysm secondary to port placement during a robot-assisted laparoscopic radical cystectomy. J Ultrasound 2020; 11: $1-4$

Ziegler MM. Diagnosis of inguinal hernia and hydrocele. Pediatric Rev 1994; 15: 286-288. 